\title{
Publisher Correction: Dysregulation of the epigenetic landscape of normal aging in Alzheimer's disease
}

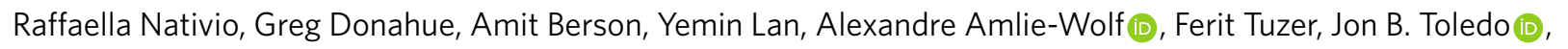
Sager J. Gosai, Brian D. Gregory, Claudio Torres, John Q. Trojanowski, Li-San Wang, F. Brad Johnson (D), Nancy M. Bonini (D) and Shelley L. Berger (D)

Correction to: Nature Neuroscience https://doi.org/10.1038/s41593-018-0101-9, published online 05 March 2018.

In the version of this article initially published online, the fifth author's name was given as Alexander Amlie-Wolf. The correct name is Alexandre Amlie-Wolf. The error has been corrected in the print, PDF and HTML versions of this article.

Published online: 19 March 2018

https://doi.org/10.1038/s41593-018-0124-2

\section{Publisher Correction: Genome-wide association study of delay discounting in 23,217 adult research participants of European ancestry}

Sandra Sanchez-Roige, Pierre Fontanillas, Sarah L. Elson, the 23andMe Research Team, Anita Pandit, Ellen M. Schmidt, Johanna R. Foerster, Gonçalo R. Abecasis, Joshua C. Gray, Harriet de Wit, Lea K. Davis (D), James MacKillop (D) and Abraham A. Palmer (iD

Correction to: Nature Neuroscience https://doi.org/10.1038/s41593-017-0032-x, published online 11 December 2017.

In the version of this article initially published, the consortium authorship was not presented correctly. The 23andMe Research Team was listed as the last author, rather than the fourth, and a line directing readers to the Supplementary Note for a list of members did appear but was not directly associated with the consortium name. Also, the Supplementary Note description stated that both member names and affiliations were included; in fact, only names are given. Finally, the URL for S-PrediXcan was given in the Methods as https://github.com/hakyimlab/S-PrediXcan; the correct URL is https:/github.com/hakyimlab/MetaXcan. The errors have been corrected in the HTML and PDF versions of the article.

\section{Publisher Correction: Viewpoints: how the hippocampus contributes to memory, navigation and cognition}

John Lisman, György Buzsáki, Howard Eichenbaum, Lynn Nadel, Charan Ranganath and A David Redish

Correction to: Nature Neuroscience https://doi.org/10.1038/nn.4661 (2017); published online 26 October 2017.

In the version of this article initially published, author Charan Ranganath's last name was misspelled Rangananth in the author list. Also, A. David Redish (redish@umn.edu) has been added as a corresponding author. The error has been corrected, and the corresponding author added, in the HTML and PDF versions of the article. 